\title{
Takotsubo Endocrinopathy
}

\author{
Sanjay Kalra, ${ }^{1}$ Om J Lakhani ${ }^{2}$ and Sandeep Chaudhary ${ }^{3}$ \\ 1. Department of Endocrinology, Bharti Hospital, Karnal, India; 2. Department of Endocrinology, Zydus Hospital, Ahmedabad, India; \\ 3. Department of Endocrinology, NMC Specialty Hospital, Dubai, United Arab Emirates
}

DOI: https://doi.org/10.17925/EE.2020.16.2.97

$\mathrm{T}$ akotsubo cardiomyopathy (TCMP) is a cardiac disorder, often seen in post-menopausal women, that resembles an acute coronary syndrome in its clinical presentation. The aetiopathogenesis of TCMP may have an endocrine basis, and hence we believe the term 'takotsubo endocrinopathy' may be more appropriate. In this review, we describe the various endocrine disorders that may lead to TCMP. We also describe the pathogenetic mechanism by which these endocrine disorders may lead to TCMP. Cardiomyopathy associated with pheochromocytoma closely resembles TCMP and we have suggested that it must be ruled out in all patients presenting with TCMP. The role of oestrogen deficiency in the pathogenesis of TCMP is examined in this article. The importance of the involvement of an endocrinologist in the management of TCMP is emphasised.

\section{Keywords}

Acute heart failure, acute coronary syndrome, adrenal glands, cardiac autonomic neuropathy, diabetes, thyroid, pheochromocytoma

Disclosure: Sanjay Kalra, Om J Lakhani and Sandeep Chaudhary have no financial or non-financial relationships or activities to declare in relation to this article. Sanjay Kalra is a member

of the journal's Editorial Board.

Review Process: Double-blind peer review.

Compliance with Ethics: This article involves a review of the literature and did not involve any studies with human or animal subjects performed by any of the authors.

Authorship: The named authors meet the Internationa Committee of Medical Journal Editors (ICMJE) criteria for authorship of this manuscript, take responsibility for the integrity of the work as a whole, and have given final approval for the version to be published.

Access: This article is freely accessible at

touchENDOCRINOLOGY.com

C) Touch Medical Media 2020

Received: 17 April 2020

Accessed: 24 June 2020

Published Online: 2 October 2020

Citation: European Endocrinology. 2020;16(2):97-9

Corresponding Author: Om J Lakhani, C-3,

1st Floor, Zydus Hospital, Thaltej, Ahmedabad,

Gujarat 380054, India. E: dromlakhani@gmail.com

Support: No funding was received in

the publication of this article.
Takotsubo cardiomyopathy (TCMP), also known as 'broken heart syndrome' or 'stress cardiomyopathy', is a well-characterised condition. It presents in the acute phase with clinical, biochemical and electrocardiographic features similar to acute myocardial infarction. However, on performing an angiogram, the patient is found to have no coronary artery involvement. 'Takotsubo' is a Japanese word for an octopus fishing trap that has a narrow opening and a wide bottom. TCMP gets its name from this because of the characteristic wall motion abnormality observed, which resembles the Japanese fishing contraption.

\section{An endocrine perspective}

TCMP is often precipitated by emotional and physical stress, and it is generally self-resolving with supportive care. Catecholamine excess and/or abnormal response of the cardiac myocytes to surge of catecholamines is thought to be the predominant pathophysiology leading to TCMP. The mortality associated with TCMP during the acute phase is similar to an episode of acute coronary syndrome. Upon follow-up, many of these patients go on to have a recurrence of TCMP and/or have increased cardiovascular mortality.

As endocrinologists, we understand that hormones do not act alone, but in concert, with one hormone impacting the clinical manifestations of others. A parallel can be drawn with diabetes mellitus. The unifying hypothesis for all forms of diabetes mellitus is absolute or relative insulin deficiency with or without insulin resistance. ${ }^{2}$ It is well known that other hormones may contribute to the development of diabetes mellitus. For example, glucocorticoid excess, as seen in Cushing's syndrome, can lead to a secondary form of diabetes. Hence, insulin alone is not responsible for the pathophysiology of diabetes mellitus. ${ }^{3}$

In the same way, TCMP can be considered a form of endocrine dysfunction of absolute or relative catecholamine surge and/or abnormal response of cardiac myocytes to a catecholamine surge impacting the heart. Other hormones, such as oestrogen, thyroid hormones and cortisol, may be either directly responsible for the surge or can modify the impact of the surge on cardiac myocytes. ${ }^{4}$ The role of various hormones in the aetiology of TCMP has been portrayed in a diagram (Figure 1). Considering this unifying hypothesis of the role of catecholamine physiology in TCMP and the long-term prognostic implications of the disease, we propose that the term 'takotsubo endocrinopathy' or 'endocrine cardiomyopathy' may be more appropriate for the disease. TCMP is known to recur and has been found to have increased short-term and long-term mortality. Keeping this in mind, the underlying endocrine disorder, which is potentially impacting the disease, needs to be recognised and treated. ${ }^{1}$

\section{Pheochromocytoma and takotsubo cardiomyopathy}

The presence of pheochromocytoma is a criterion for exclusion of TCMP. However, heochromocytoma can present with cardiac manifestations similar to TCMP, due to a surge of catecholamines. ${ }^{5}$ The first case resembling TCMP was described in a patient with pheochromocytoma. ${ }^{6}$ 
Figure 1: Summary of the role of various hormones in the pathogenesis of takotsubo cardiomyopathy

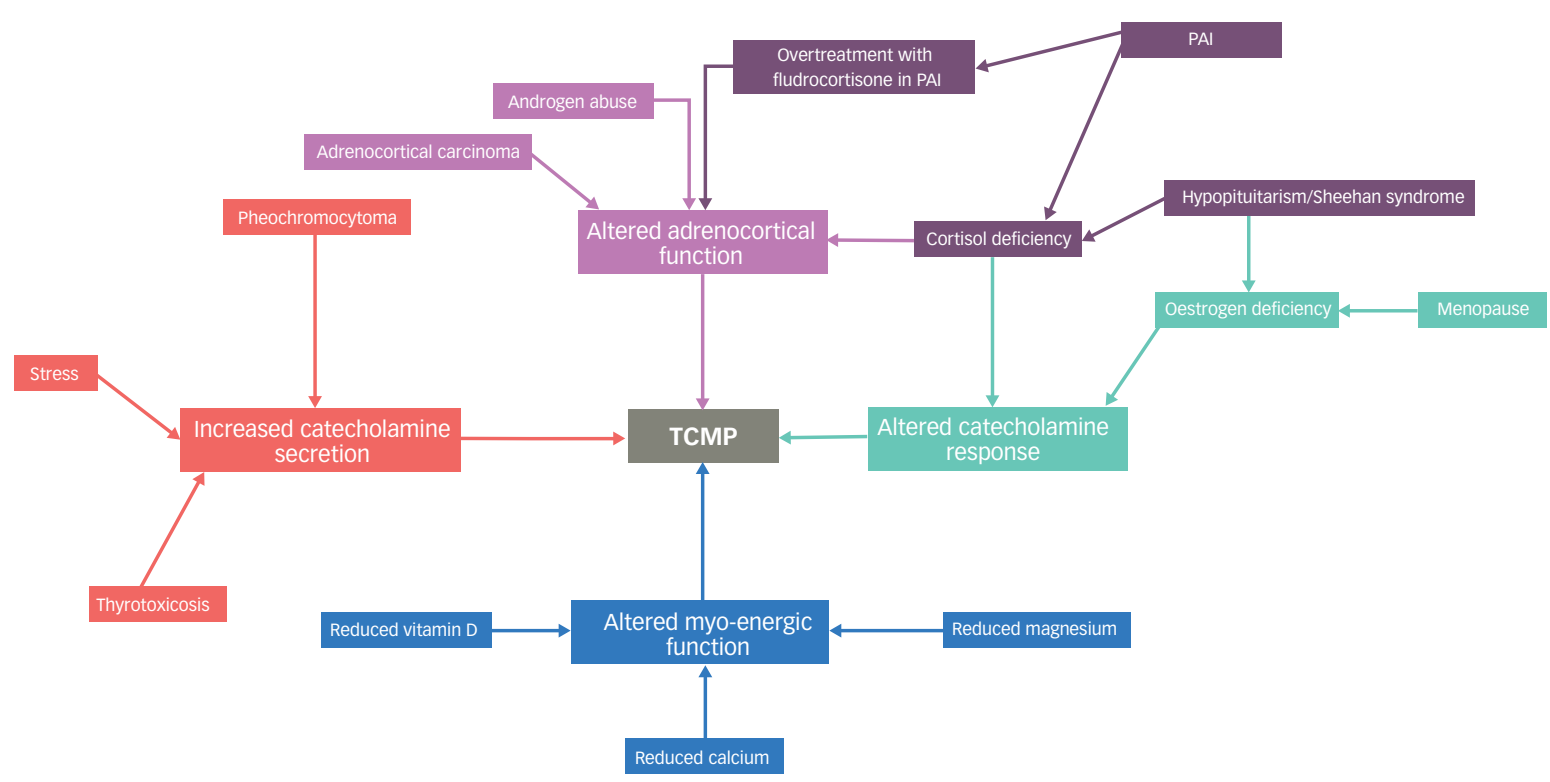

PAI = primary adrenal insufficiency; $T C M P=$ takotsubo cardiomyopathy.

Hence, pheochromocytoma must always be ruled out in patients presenting a clinical picture resembling TCMP. ${ }^{4}$

At a physiological level, adrenaline has positive inotropic and lusitropic effects, mediated by activation of the adenylyl cyclase protein kinase A pathway. At 'supraphysiological' levels, however, adrenaline switches its $\beta_{2}$ adrenoreceptor coupling from $G_{s}$ protein to $G_{i}$ protein signalling in both atrial and ventricular muscles. This seemingly adaptive response leads to negative inotropism and may protect the heart from extreme stress.' Noradrenaline, on the other hand, continues to exhibit positive inotropism through its preferential binding of the $\beta_{1}$ adreno receptors. The ratio of $\beta_{2}: \beta_{1}$ adrenoreceptors is $1: 4$ in all parts of the ventricular myocardium, except the apex, where $\beta_{2}$ receptors are denser. ${ }^{7}$ It is possible that predominant $\mathrm{G}_{i}$ signalling in the apex can cause the ampulla-shaped topography so typical of TCMP. ${ }^{8}$ However, apical ballooning is not always observed in all patients, and hence this theory may not hold true for other forms of TCMP.

Though pheochromocytoma is proposed as an exclusion criterion for diagnosis of TCMP, we believe that the cardiomyopathy associated with pheochromocytoma and TCMP should be considered the same disease. Pheochromocytoma should be ruled out in all patients presenting with TCMP. ${ }^{6}$ This reinforces the critical role played by endocrinologist in patients presenting with TCMP.

\section{Takotsubo cardiomyopathy associated with thyrotoxicosis}

Catecholamines play a crucial rule in the cardiac manifestation of thyrotoxicosis. In a review of published cases of TCMP, De Girogi et al. reported that thyrotoxicosis is the most common endocrine disorder associated with TCMP. TCMP has been associated with newly diagnosed thyrotoxicosis, including patients presenting with thyroid storm and also with radioactive iodine ablation of hyperthyroidism. Hence it is important to rule out thyrotoxicosis in patients presenting with TCMP. We believe that a thyroid function test should be performed in all patients with TCMP.4
The role of the endocrinologist in the diagnosis and treatment of pheochromocytoma and thyrotoxicosis in patients presenting with TCMP is clear. However, patients with TCMP may not have either of these conditions. Therefore, it is important to explore the role of other endocrine disorders in the aetiopathogenesis of TCMP.

\section{The role of oestrogen deficiency in the pathophysiology of takotsubo cardiomyopathy}

TCMP is most often noted in post-menopausal women. Reduced oestrogen has been thought to increase the vulnerability of cardiac myocytes to sudden changes in catecholamine levels.' Genetic studies have suggested a link between oestrogen receptor polymorphisms and the risk of TCMP. The highest risk is noted in women carrying a T allele at the rs1271572 locus of the oestrogen receptor 2 gene. ${ }^{10} \mathrm{~A}$ study conducted in ovariectomised female rats has shown that 2 weeks of oestradiol supplementation reduced mortality in rats with TCMP-like cardiac dysfunction. ${ }^{11}$ Another study has shown that oestradiol helps protect against the toxic effects of catecholamines on cardiac myocytes. ${ }^{12}$ The role of endocrine manipulation in reducing mortality and preventing the recurrence of TCMP in post-menopausal women needs to be explored in a dedicated study.

\section{The role of cortisol deficiency in precipitating takotsubo cardiomyopathy}

TCMP can be precipitated by acute adrenal crisis due to isolated adrenocorticotropic hormone (ACTH) deficiency. ${ }^{13}$ A similar case has also been seen in central adrenal insufficiency due to pituitary adenoma. ${ }^{11}$ Sheehan syndrome is a post-partum panhypopituitarism caused by peripartum haemorrhage and is widely reported in developing countries. Case reports have shown an association of Sheehan syndrome with a reversible cardiomyopathy reminiscent of TCMP. ${ }^{15}$ Central adrenal insufficiency combined with central hypothyroidism may be the aetiological cause of cardiomyopathy associated with Sheehan syndrome. ${ }^{4}$ We believe that this reversible cardiomyopathy seen in Sheehan syndrome could be a form of TCMP. 


\section{The relationship between diabetes mellitus and takotsubo cardiomyopathy}

Surprisingly, a 'diabetes paradox' has been reported in TCMP.' Microvascular angiopathy and diabetic autonomic neuropathy have been implicated in the pathogenesis of TCMP. ${ }^{17}$ However, the prevalence of diabetes mellitus in people with TCMP appears to be lower than normal. ${ }^{18}$ This may be due to a 'cerebro-cardiac disconnect' which attenuates the effects of catecholamine surge on the heart.19 It is possible that this may be a protective manifestation of cardiac autonomic neuropathy. Recent reports from multicentric registries, however, report that diabetes is common in TCMP, and is associated with adverse outcomes including lower long-term survival. ${ }^{20}$ The 'cerebro-cardiac disconnect' continues to be postulated as an explanation for the lower prevalence of diabetes observed. It is possible that the wide spectrum of cardiac autonomic neuropathy and TCMP may overlap in different ways. More work is required to elucidate the mechanisms of this interaction.

\section{The role of other hormones and endocrine disorders in takotsubo cardiomyopathy}

Vitamin $\mathrm{D}$ insufficiency has been reported to be more prevalent in patients presenting with TCMP. Vitamin D insufficiency is also associated with worse haemodynamic parameters in affected women. ${ }^{21}$ It is possible that lack of Vitamin D leads to suboptimal myocardial reserve, which predisposes to cardiac decompensation in the face of stressors. This pathogenesis may be similar to that of reversible dilated cardiomyopathy encountered in severe rickets in children. ${ }^{21}$

Rhee et al. have described a case in which TCMP was found to be associated with severe hypocalcemia secondary to idiopathic hypoparathyroidism. The symptoms and the cardiac function improved in this patient with correction of the hypocalcemia using calcium supplements and calcitriol.22

TCMP has been noted to be associated with autoimmune polyendocrine syndrome type II (Schmidt's syndrome). ${ }^{23}$ This syndrome is characterised by Addison's disease with either or both type 1 diabetes mellitus and autoimmune thyroid disease. ${ }^{6}$ Cases of TCMP have been known to be triggered by fludrocortisone overdose for Addison's disease. This decompensation may be due to increased myocardial vulnerability due to cortisol deficiency, which is triggered by an overdose of fludrocortisones. ${ }^{24}$ This information suggests the need for gradual and slow titration while managing Addison's disease, along with heightened 'cardiovigilance'.

While there are no reports of testosterone-induced TCMP available, Placci et al. described a 25-year-old body builder who developed TCMP due to stanozolol and nandrolone abuse. ${ }^{25}$ It is possible that androgens may have a protective regulatory role on the cardiomyocytes. There is no definite evidence, however, that exogenous administration of testosterone improves cardiovascular health.

\section{Practical implications}

Takotsubo syndrome, whether named 'takotsubo cardiomyopathy' or as 'takotsubo endocrinopathy' is associated with multiple endocrine aetiologies. All cardiologists should be aware of this, and should practice 'endovigilance' while managing these patients. It must be remembered that endocrine deficiency, and hormonal excess (whether endogenous or iatrogenic), may precipitate TCMP (Figure 1). Endocrine consultation should be sought to screen, diagnose and manage associated endocrine comorbidity wherever necessary. At the same time, endocrinologists should be cognisant of the possible cardiac manifestation of uncontrolled endocrine disorders. Cardiovigilance while managing endocrine disease, with timely referral for cardiovascular support, will help prevent avoidable complications

\section{Conclusions}

TCMP is increasingly being recognised as an important cause of acute heart failure and a close mimic of acute coronary syndrome. Several endocrine disorders are found to be potentially associated with TCMP or reversible forms of cardiomyopathy similar to TCMP. Since TCMP is known to recur and is found to have increased short-term and long-term mortality, diagnosis of the underlying endocrine disorder needs to be recognised and treated. This implies that endocrinologists and cardiologists need to work as a team to prevent and manage this syndrome. $\square$
1. Templin C, Ghadri JR, Diekmann I, et al. Clinical features and outcomes of takotsubo (stress) cardiomyopathy. $N$ Eng/ I Med. 2015:373:929-38

2. Thévenod F. Pathophysiology of diabetes mellitus type 2: roles of obesity, insulin resistance and $\beta$-cell dysfunction. In: Masur K, Thévenod F, Zänker KS (eds). Diabetes and Cancer. Basel: Karger Publishers, 2008;19:1-18

3. Pivonello R, De Leo M, Vitale P, et al. Pathophysiology of diabetes mellitus in Cushing's syndrome. Neuroendocrinology. 2010;92(Suppl. 1):77-81.

4. Gupta S, Goyal P, Idrees S, et al. Association of endocrine conditions with Takotsubo cardiomyopathy: a comprehensive review. J Am Heart ASSOC. 2018;7:1-19.

5. Prasad A, Lerman A, Rihal CS. Apical ballooning syndrome (tako-tsubo or stress cardiomyopathy): a mimic of acute myocardial infarction. Am Heart J. 2008:155:408-17.

6. Iga K, Gen H, Tomonaga G, et al. Reversible left ventricular wall motion impairment caused by pheochromocytoma: a case report. Jpn Circ J. 1989:53:813-8.

7. Lyon AR, Rees PS, Prasad S, et al. Stress (takotsubo) cardiomyopathy - a novel pathophysiological hypothesis to explain catecholamine-induced acute myocardial stunning. Nat Clin Pract Cardiovasc Med. 2008;5:22-9.

8. Gujja KR, Aslam AF, Privman V, et al. Initial presentation of pheochromocytoma with takotsubo cardiomyopathy: A brief review of literature. J Cardiovasc Med (Hagerstown). 2020;11:49-52.

9. De Giorgi A, Fabbian F, Tiseo R, et al. Takotsubo cardiomyopathy and endocrine disorders: A mini-review of case reports. Am J Emerg Med. 2014:32:1413-7.

10. Pizzino $G$, Bitto A, Crea P, et al. Takotsubo syndrome and estrogen receptor genes: Partners in crime? J Cardiovasc Med (Hagerstown) 2017:18:268-76.

11. Tranter M, Wright P, Lyon A, Harding S. P763 Ovariectomy increases epinephrine-induced mortality in a rat takotsubo cardiomyopathy model: the effects of estrogen supplementation. Cardiovasc Res. 2014;103(Suppl. 1):S140

12. El-Battrawy I, Zhao Z, Lan H, et al. Estradiol protection against toxic effects of catecholamine on electrical properties in human-induced pluripotent stem cell derived cardiomyocytes. Int J Cardiol. 2018;254:195-202.

13. Ukita C, Miyazaki H, Toyoda N, et al. Takotsubo cardiomyopathy during acute adrenal crisis due to isolated adrenocorticotropin deficiency. Intern Med. 2009:48:347-52.

14. Singh G, Manickam A, Sethuraman M, Rathod R. Takotsubo cardiomyopathy in a patient with pituitary adenoma and secondary adrenal insufficiency. Indian I Crit Care Med. secondary adr

15. Lee SH, Kim KJ, Kim BY, et al. A case of stress-induced cardiomyopathy in Sheehan's syndrome. Korean J Med. 2014;87:347-51

16. Bill V, El-Battrawy I, Behnes M, et al. "Diabetes paradox" in takotsubo cardiomyopathy. Int J Cardiol. 2016;224:88-9.

7. Khalid N, Ahmad SA, Umer A, Chhabra L. Role of microcirculatory disturbances and diabetic autonomic neuropathy in takotsubo cardiomyopathy. Crit Care Med. 2015;43:e527

18. Madias JE Low prevalence of diabetes mellitus in patients with takotsubo syndrome: A plausible "protective" effect with pathophysiologic connotations. Eur Heart I Acute Cardiovasc Care 2016:5:164-70.

19. Madias JE. Diabetes mellitus prevalence in patients with takotsubo syndrome: The case of the brain-heart disconnect. Heart Lung. 2018;47:222-5.

20. Stiermaier T, Santoro F, El-Battrawy I, et al. Prevalence and prognostic impact of diabetes in takotsubo syndrome: Insights from the international, multicenter GEIST registry. Diabetes Care. 2018;41:1084-8.

21. Dande AS, Sena SF, Wasserman HS, et al. Prevalence and consequences of vitamin D insufficiency in women with takotsubo cardiomyopathy. J Clin Endocrinol Metab. 2013;98:E872-6

22. Rhee HS, Lee SW, Jung YK, et al. Takotsubo cardiomyopathy associated with severe hypocalcemia secondary to idiopathic hypoparathyroidism. Korean Circ I. 2013:43:573-7.

23. Lim T, Murakami H, Hayashi K, et al. Takotsubo cardiomyopathy associated with autoimmune polyendocrine syndrome II. Cardiol. 2009;53:306-10.

24. Campean R, Hasun M, Stöllberger C, et al. Takotsubo-like syndrome triggered by fludrocortisone overdose for Addison's disease: a case report. J Med Case Rep. 2016;10:281.

25. Placci A, Sella G, Bellanti G, Margheri M. Anabolic androgenic steroid-induced takotsubo cardiomyopathy. BMJ Case Rep. 2015;2015:bcr2014209089 